\title{
Phase and Antiphase Synchronization between 3-Cell CNN and Volta Fractional-Order Chaotic Systems via Active Control
}

\author{
Zahra Yaghoubi and Hassan Zarabadipour \\ Department of Electrical Engineering, Imam Khomeini International University, Qazvin 34149-16818, Iran \\ Correspondence should be addressed to Zahra Yaghoubi, mn_y3567@yahoo.com
}

Received 23 November 2011; Revised 10 February 2012; Accepted 12 February 2012

Academic Editor: Ahmad M. Harb

Copyright (c) 2012 Z. Yaghoubi and H. Zarabadipour. This is an open access article distributed under the Creative Commons Attribution License, which permits unrestricted use, distribution, and reproduction in any medium, provided the original work is properly cited.

Synchronization of fractional-order chaotic dynamical systems is receiving increasing attention owing to its interesting applications in secure communications of analog and digital signals and cryptographic systems. In this paper, a drive-response synchronization method is studied for "phase and antiphase synchronization" of a class of fractional-order chaotic systems via active control method, using the 3-cell and Volta systems as an example. These examples are used to illustrate the effectiveness of the synchronization method.

\section{Introduction}

The theory of fractional calculus is a 300-year-old topic which can trace back to Leibniz, Riemann, Liouville, Grünwald, and Letnikov [1,2]. However, the fractional calculus did not attract much attention for a long time. Nowadays, the past three decades have witnessed significant progress on fractional calculus, because the applications of fractional calculus were found in more and more scientific fields, covering mechanics, physics, engineering, informatics, and materials. Nowadays, it has been found that some fractionalorder differential systems such as the fractional-order jerk model [3], the fractional-order Rössler system [4], and the fractional-order Arneodo system [5] can demonstrate chaotic behavior.

Recently, synchronization of fractional-order chaotic systems has started to attract increasing attention due to its potential applications in secure communication and control processing [6, 7]. The concept of synchronization can be extended to generalized synchronization [8], complete synchronization [9], lag synchronization [10], phase synchronization, antiphase synchronization [11], and so on. 
Synchronization of fractional-order chaotic systems was first studied by Deng and Li [12] who carried out synchronization in case of the fractional Lü system. Further, they have investigated synchronization of fractional Chen system [13].

In this paper, phase and anti-phase synchronization using is introduced, which is used to "phase and anti-phase synchronization" for a class of fractional-order chaotic systems using active control method [14].

The outline of the rest of the paper is organized as follows. First, Section 2 provides a brief review of the fractional derivative and the numerical algorithm of fractional-order differential equation. Section 3 is devoted to 3-Cell and Volta systems description. Next, in Section 4, the definition of phase and anti-phase synchronization is introduced. In Section 5 , the proposed method is applied to synchronize two examples of fractional-order chaotic systems. Finally, Section 6 is the brief conclusion.

\section{Fractional Derivative and Numerical Algorithm of Fractional Differential Equation}

There are many definitions of fractional derivatives $[15,16]$. Many authors formally use the Riemann-Liouville fractional derivatives, defined by

$$
D^{\alpha} x(t)=\frac{d^{m}}{d t^{m}} J^{m-\alpha} x(t), \quad \alpha>0
$$

where $m=[\alpha]$, that is, $m$ is the first integer which is not less than $\alpha \cdot J^{\beta}$ is the $\beta$-order RiemannLiouville integral operator, and $\Gamma(\cdot)$ is the gamma function which is described as follows:

$$
\begin{gathered}
J^{\alpha} x(t)=\frac{1}{\Gamma(\alpha)} \int_{0}^{t}(t-\tau)^{\alpha-1} f(\tau) d \tau \\
\Gamma(z)=\int_{0}^{\infty} t^{z-1} e^{-t} d t .
\end{gathered}
$$

In this paper, the following definition is used:

$$
D_{*}^{\alpha} x(t)=J^{m-\alpha} x^{(m)}(t), \quad \alpha>0 .
$$

[17].

It is common practice to call operator $D_{*}^{\alpha}$ the Caputo differential operator of order $\alpha$

The numerical calculation of a fractional differential equation is not so simple as that of an ordinary differential equation. Here, we choose the Caputo version and use a predictorcorrector algorithm for fractional differential equations [18], which is the generalization of Adams-Bashforth-Moulton one. When $\alpha>0$, the algorithm is universal. The following is a brief introduction of the algorithm. The differential equation

$$
\begin{gathered}
D_{*}^{\alpha} x(t)=f(t, x(t)), \quad 0 \leq t \leq T, \\
x^{(k)}(0)=x_{0}^{(k)}, \quad k=0,1, \ldots, m-1 .
\end{gathered}
$$




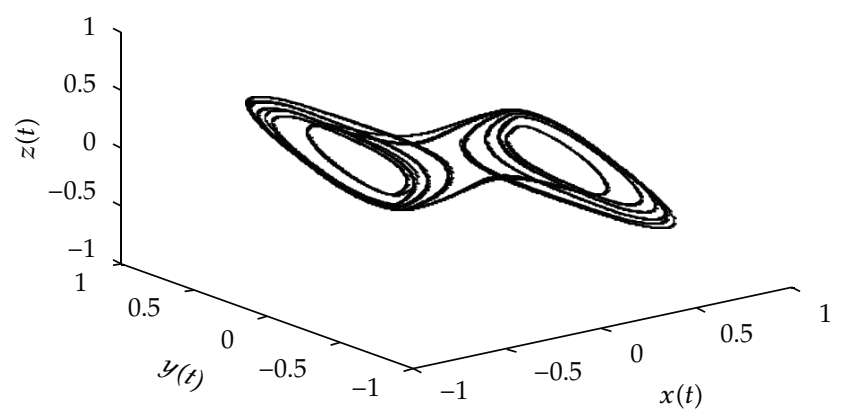

Figure 1: Chaotic attractor of 3-cell CNN's system (3.1).

is equivalent to the Volterra integral equation

$$
x(t)=\sum_{k=0}^{[a]-1} \frac{t^{k}}{k !} x_{0}^{(k)}+\frac{1}{\Gamma(\alpha)} \int_{0}^{t}(t-\tau)^{\alpha-1} f(\tau, x(\tau)) d \tau .
$$

\section{Systems Description}

Chua and Yang introduced the cellular neural network (CNN) in 1988 as a nonlinear dynamical system composed by an array of elementary and locally interacting nonlinear subsystems, so called cells [19].

Arena et al. introduced a new class of the $\mathrm{CNN}$ with fractional- (noninteger-) order cells [20].

Hartley et al. introduced a fractional-order Chua's system [21]. From this consideration, the idea of developing a fractional-order $\mathrm{CNN}$ arose. This system is described as follows:

$$
\begin{aligned}
& D^{\alpha} x(t)=-x(t)+p_{1} f(x(t))-s f(y(t))-s f(z(t)), \\
& D^{\alpha} y(t)=-y(t)-s f(x(t))+p_{2} f(y(t))-r f(z(t)), \\
& D^{\alpha} z(t)=-z(t)-s f(x(t))+r f(y(t))+p_{3} f(z(t)),
\end{aligned}
$$

where $f(x)=0.5(|x+1|-|x-1|)$. In Figure 1 is shown the chaotic behavior for fractionalorder chaotic system (3.1), where system parameters are $p_{1}=1.24, s=3.21, p_{2}=1.1, r=4.4$, and $p_{3}=1$, commensurate order of the derivatives is $\alpha=0.99$, and the initial conditions are $x(0)=0.1, y(0)=0.1$, and $z(0)=0.1$ for the simulation time $T_{\mathrm{sim}}=100 \mathrm{~s}$ and time step $h=0.005$.

Petráš [22, 23] has pointed out that system (3.2) shows chaotic behavior for suitable $a$, $b$, and $c$. Fractional-order Volta system can be written in the form of (3.2) as

$$
\begin{aligned}
& D^{\alpha} x(t)=-x(t)-a y(t)-z(t) y(t), \\
& D^{\alpha} y(t)=-y(t)-b x(t)-x(t) z(t), \\
& D^{\alpha} z(t)=c z(t)+x(t) y(t)+1 .
\end{aligned}
$$




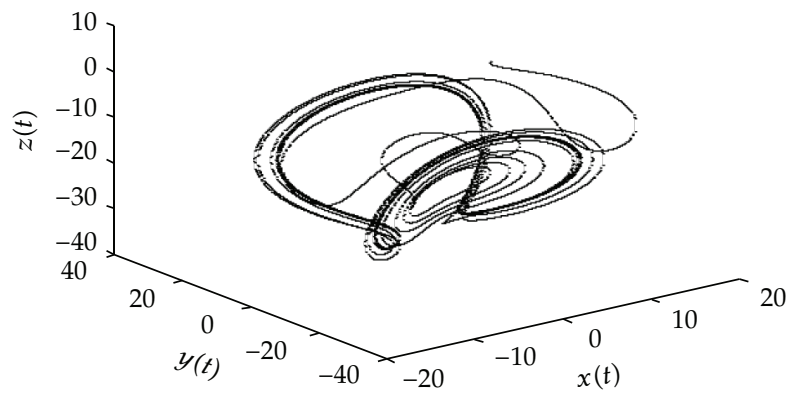

Figure 2: Chaotic attractor of Volta's system (3.2).

In Figure 2 is shown the chaotic behavior for fractional-order chaotic system (3.2), where system parameters are $a=19, b=11$, and $c=0.73$, commensurate order of the derivatives is $\alpha=0.99$, and the initial conditions are $x(0)=8, y(0)=2$, and $z(0)=1$ for the simulation time $T_{\text {sim }}=20 \mathrm{~s}$ and time step $h=0.0005$.

\section{Phase Synchronization}

In this section, we study the phase synchronization between the two fractional-order 3-cell CNN and Volta systems by means of active control.

Consider 3-cell CNN system as the drive system

$$
\begin{aligned}
& D^{\alpha} x_{1}(t)=-x_{1}(t)+p_{1} f\left(x_{1}(t)\right)-s f\left(y_{1}(t)\right)-s f\left(z_{1}(t)\right), \\
& D^{\alpha} y_{1}(t)=-y_{1}(t)-s f\left(x_{1}(t)\right)+p_{2} f\left(y_{1}(t)\right)-r f\left(z_{1}(t)\right), \\
& D^{\alpha} z_{1}(t)=-z_{1}(t)-s f\left(x_{1}(t)\right)+r f\left(y_{1}(t)\right)+p_{3} f\left(z_{1}(t)\right) .
\end{aligned}
$$

and Volta system as the response system

$$
\begin{aligned}
& D^{\alpha} x_{2}(t)=-x_{2}(t)-a y_{2}(t)-z_{2}(t) y_{2}(t)+u_{1}(t), \\
& D^{\alpha} y_{2}(t)=-y_{2}(t)-b x_{2}(t)-x_{2}(t) z_{2}(t)+u_{2}(t), \\
& D^{\alpha} z_{2}(t)=c z_{2}(t)+x_{2}(t) y_{2}(t)+1+u_{3}(t) .
\end{aligned}
$$

Define the error functions as $e_{1}=x_{2}-x_{1}, e_{2}=y_{2}-y_{1}$, and $e_{3}=z_{2}-z_{1}$. For phase synchronization, it is essential that the errors $e_{i}$ tend to a zero as $t \rightarrow \infty$. In order to determine the control functions $u_{i}$, we subtract (4.1) from (4.2) and obtain

$$
\begin{aligned}
& D^{\alpha} e_{1}(t)=-e_{1}-a e_{2}-a y_{1}-e_{3} e_{2}-z_{1} e_{2}-e_{3} y_{1}-z_{1} y_{1}-p_{1} f\left(x_{1}\right)+s f\left(y_{1}\right)+s f\left(z_{1}\right)+u_{1}(t), \\
& D^{\alpha} e_{2}(t)=-e_{2}-b e_{1}-b x_{1}-e_{1} z_{1}-x_{1} z_{1}-e_{1} e_{3}-x_{1} e_{3}+s f\left(x_{1}\right)-p_{2} f\left(y_{1}\right)+r f\left(z_{1}\right)+u_{2}(t), \\
& D^{\alpha} e_{3}(t)=1+c e_{3}+c z_{1}+e_{1} e_{2}+x_{1} e_{2}+e_{1} y_{1}+x_{1} y_{1}+z_{1}+s f\left(x_{1}\right)-r f\left(y_{1}\right)-p_{3} f\left(z_{1}\right)+u_{3}(t) .
\end{aligned}
$$


Choosing the control functions

$$
\begin{aligned}
& u_{1}(t)=a y_{1}+e_{3} e_{2}+z_{1} e_{2}+e_{3} y_{1}+z_{1} y_{1}+p_{1} f\left(x_{1}\right)-s f\left(y_{1}\right)-s f\left(z_{1}\right)+V_{1}(t) \\
& u_{2}(t)=b x_{1}+e_{1} z_{1}+x_{1} z_{1}+e_{1} e_{3}+x_{1} e_{3}-s f\left(x_{1}\right)+p_{2} f\left(y_{1}\right)-r f\left(z_{1}\right)+V_{2}(t) \\
& u_{3}(t)=-c z_{1}-e_{1} e_{2}-x_{1} e_{2}+e_{1} y_{1}-x_{1} y_{1}-z_{1}-s f\left(x_{1}\right)+r f\left(y_{1}\right)+p_{3} f\left(z_{1}\right)+V_{3}(t)
\end{aligned}
$$

Equation (4.3) leads to

$$
\begin{aligned}
& D^{\alpha} e_{1}(t)=-e_{1}-a e_{2}+V_{1}(t), \\
& D^{\alpha} e_{2}(t)=-e_{2}-b e_{1}+V_{2}(t), \\
& D^{\alpha} e_{3}(t)=1-c e_{3}+V_{3}(t) .
\end{aligned}
$$

The linear functions $V_{1}, V_{2}$, and $V_{3}$ are given by

$$
\begin{aligned}
& V_{1}(t)=e_{1}+a e_{2}+\lambda_{1} e_{1}, \\
& V_{2}(t)=e_{2}+b e_{1}+\lambda_{2} e_{2}, \\
& V_{3}(t)=-1+c e_{3}+\lambda_{3} e_{3},
\end{aligned}
$$

where $\lambda_{1}, \lambda_{2}$, and $\lambda_{3}$ are the eigenvalues of the linear system (4.5).

\subsection{Simulation Results}

Parameters of 3-cell CNN and Volta systems are $p_{1}=1.24, s=3.21, p_{2}=1.1, r=4.4$, and $p_{3}=1$ and $a=19, b=11$, and $c=0.73$, respectively. The initial conditions for drive and response systems are $x_{1}(0)=0.1, y_{1}(0)=0.1$, and $z_{1}(0)=0.1$ and $x_{2}(0)=8, y_{2}(0)=2$, and $z_{2}(0)=1$, respectively. By choosing $\left(\lambda_{1}, \lambda_{2}, \lambda_{3}\right)=(-1,-1,-1)$, the control functions can be determined, and phase synchronization between signals $\left(x_{1}, x_{2}\right),\left(y_{1}, y_{2}\right)$, and $\left(z_{1}, z_{2}\right)$ will be achieved, respectively. Numerical results are illustrated in Figures 3(a)-3(c) for fractionalorder $\alpha=0.99$. The curves of synchronization errors are shown in Figure 4 , and the phase diagrams of (4.1) and (4.2) are plotted together in Figure 5.

\section{Antiphase Synchronization}

In this section, we study the anti-phase synchronization between the two fractional-order 3-cell CNN and Volta systems by means of active control.

Consider 3-cell CNN system as the drive system

$$
\begin{aligned}
& D^{\alpha} x_{1}(t)=-x_{1}(t)+p_{1} f\left(x_{1}(t)\right)-s f\left(y_{1}(t)\right)-s f\left(z_{1}(t)\right), \\
& D^{\alpha} y_{1}(t)=-y_{1}(t)-s f\left(x_{1}(t)\right)+p_{2} f\left(y_{1}(t)\right)-r f\left(z_{1}(t)\right), \\
& D^{\alpha} z_{1}(t)=-z_{1}(t)-s f\left(x_{1}(t)\right)+r f\left(y_{1}(t)\right)+p_{3} f\left(z_{1}(t)\right) .
\end{aligned}
$$




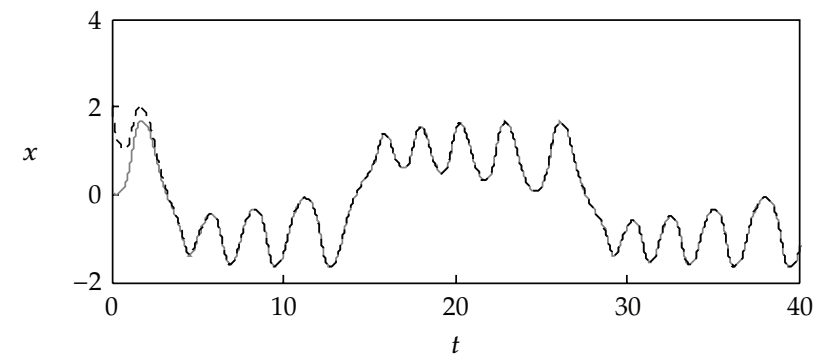

$-\begin{array}{ll}- & x_{1} \\ --- & x_{2}\end{array}$

(a)

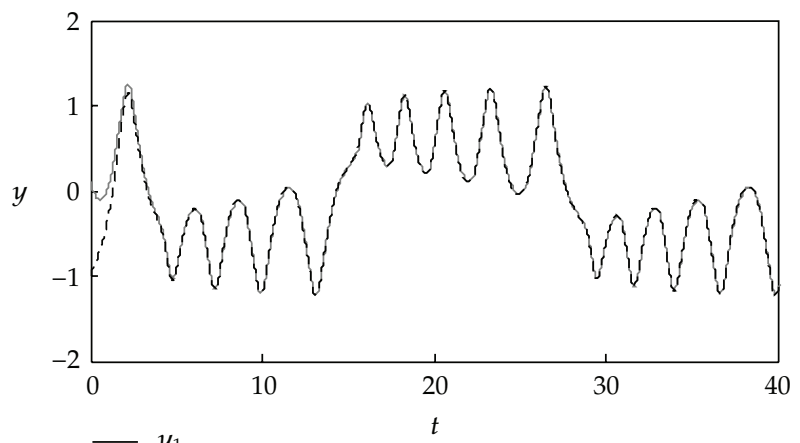

$\begin{array}{ll}- & y_{1} \\ --- & y_{2}\end{array}$

(b)

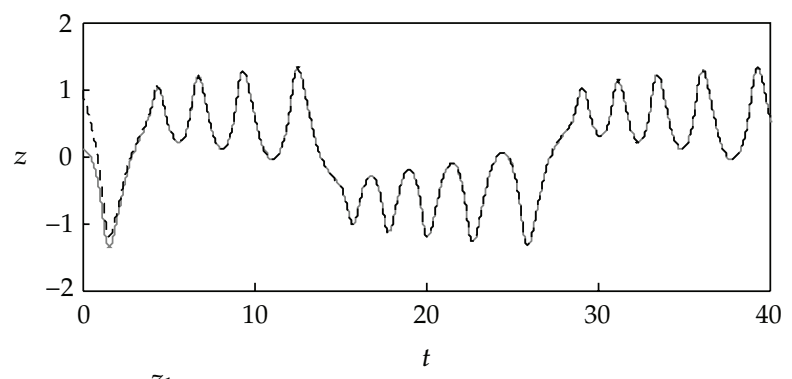

$\begin{array}{ll}- & z_{1} \\ --- & z_{2}\end{array}$

(c)

Figure 3: Phase synchronization with fractional-order $\alpha=0.99$ for signals $\left(x_{1}, x_{2}\right)$ in $(a),\left(y_{1}, y_{2}\right)$ in $(b)$, and $\left(z_{1}, z_{2}\right)$ in $(\mathrm{c})$.

and Volta system as the response system

$$
\begin{aligned}
& D^{\alpha} x_{2}(t)=-x_{2}(t)-a y_{2}(t)-z_{2}(t) y_{2}(t)+u_{1}(t), \\
& D^{\alpha} y_{2}(t)=-y_{2}(t)-b x_{2}(t)-x_{2}(t) z_{2}(t)+u_{2}(t), \\
& D^{\alpha} z_{2}(t)=c z_{2}(t)+x_{2}(t) y_{2}(t)+1+u_{3}(t) .
\end{aligned}
$$




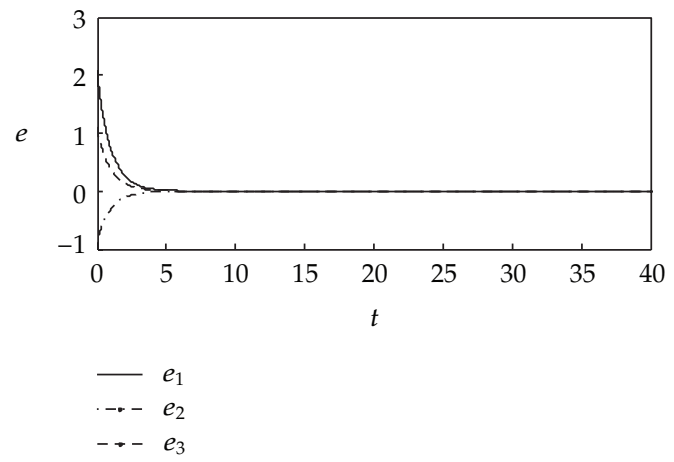

Figure 4: Synchronization errors of drive system (4.1) and response system (4.2).

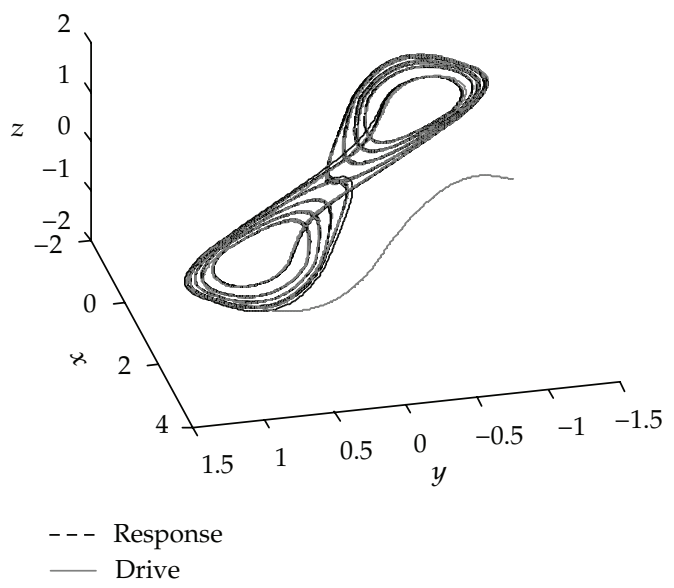

Figure 5: The attractors of drive system (4.1) and response system (4.2).

Define the error functions as $e_{1}=x_{2}+x_{1}, e_{2}=y_{2}+y_{1}$, and $e_{3}=z_{2}+z_{1}$. For phase synchronization, it is essential that the errors $e_{i}$ tend to a zero as $t \rightarrow \infty$. In order to determine the control functions $u_{i}$, we subtract (5.1) from (5.2) and obtain

$$
\begin{aligned}
& D^{\alpha} e_{1}(t)=-e_{1}-a e_{2}-a y_{1}-e_{3} e_{2}-z_{1} e_{2}-e_{3} y_{1}-z_{1} y_{1}-2 x_{1}+p_{1} f\left(x_{1}\right)-s f\left(y_{1}\right)-s f\left(z_{1}\right)+u_{1}(t), \\
& D^{\alpha} e_{2}(t)=-e_{2}-b e_{1}-b x_{1}-e_{1} z_{1}-x_{1} z_{1}-e_{1} e_{3}-x_{1} e_{3}-2 y_{1}-s f\left(x_{1}\right)+p_{2} f\left(y_{1}\right)-r f\left(z_{1}\right)+u_{2}(t), \\
& D^{\alpha} e_{3}(t)=1+c e_{3}+c z_{1}+e_{1} e_{2}+x_{1} e_{2}+e_{1} y_{1}+x_{1} y_{1}-z_{1}-s f\left(x_{1}\right)+r f\left(y_{1}\right)+p_{3} f\left(z_{1}\right)+u_{3}(t) .
\end{aligned}
$$

Choosing the control functions

$$
\begin{aligned}
& u_{1}(t)=a y_{1}+e_{3} e_{2}+z_{1} e_{2}+e_{3} y_{1}+z_{1} y_{1}+2 x_{1}-p_{1} f\left(x_{1}\right)+s f\left(y_{1}\right)+s f\left(z_{1}\right)+V_{1}(t), \\
& u_{2}(t)=b x_{1}+e_{1} z_{1}+x_{1} z_{1}+e_{1} e_{3}+x_{1} e_{3}+2 y_{1}+s f\left(x_{1}\right)-p_{2} f\left(y_{1}\right)+r f\left(z_{1}\right)+V_{2}(t), \\
& u_{3}(t)=-e_{1} e_{2}-x_{1} e_{2}-e_{1} y_{1}-x_{1} y_{1}-c z_{1}+z_{1}+s f\left(x_{1}\right)-r f\left(y_{1}\right)-p_{3} f\left(z_{1}\right)+V_{3}(t) .
\end{aligned}
$$




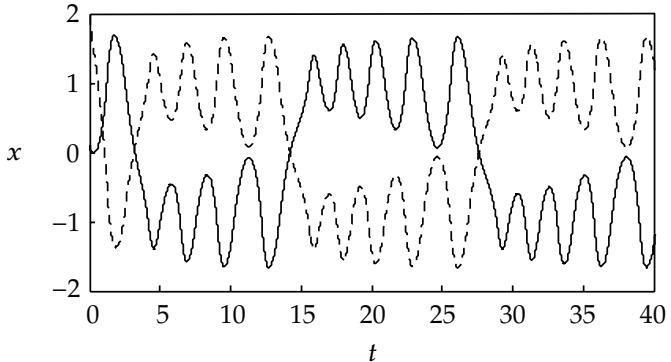

$-\begin{array}{cc}- & x_{1} \\ --- & x_{2}\end{array}$

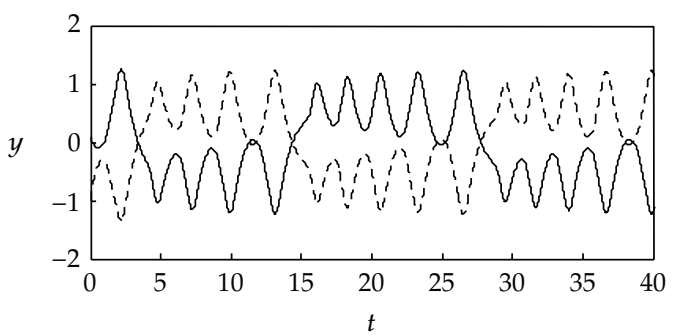

$---y_{2}$

(a)

(b)

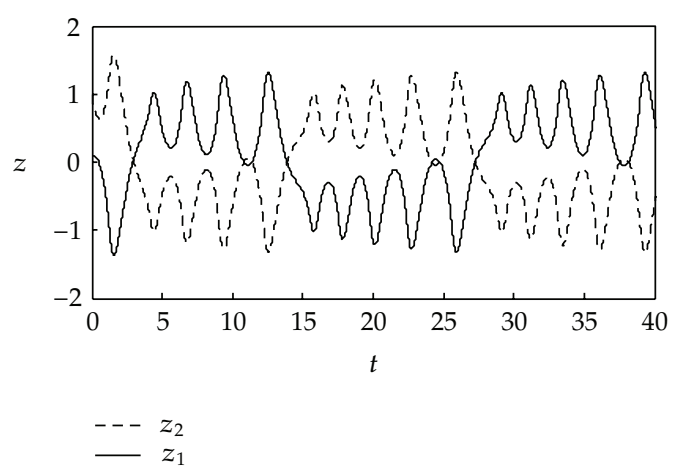

(c)

Figure 6: Antiphase synchronization with fractional-order $\alpha=0.99$ for signals $\left(x_{1}, x_{2}\right)$ in $(a),\left(y_{1}, y_{2}\right)$ in (b), and $\left(z_{1}, z_{2}\right)$ in (c).

Equation(5.3) leads to

$$
\begin{aligned}
& D^{\alpha} e_{1}(t)=-e_{1}-a e_{2}+V_{1}(t), \\
& D^{\alpha} e_{2}(t)=-e_{2}-b e_{1}+V_{2}(t), \\
& D^{\alpha} e_{3}(t)=1+c e_{3}+V_{3}(t) .
\end{aligned}
$$

The linear functions $V_{1}, V_{2}$, and $V_{3}$ are given by

$$
\begin{aligned}
& V_{1}(t)=e_{1}+a e_{2}+\lambda_{1} e_{1}, \\
& V_{2}(t)=e_{2}+b e_{1}+\lambda_{2} e_{2}, \\
& V_{3}(t)=-1-c e_{3}+\lambda_{3} e_{3},
\end{aligned}
$$

where $\lambda_{1}, \lambda_{2}$, and $\lambda_{3}$ are the eigenvalues of the linear system (5.5). 


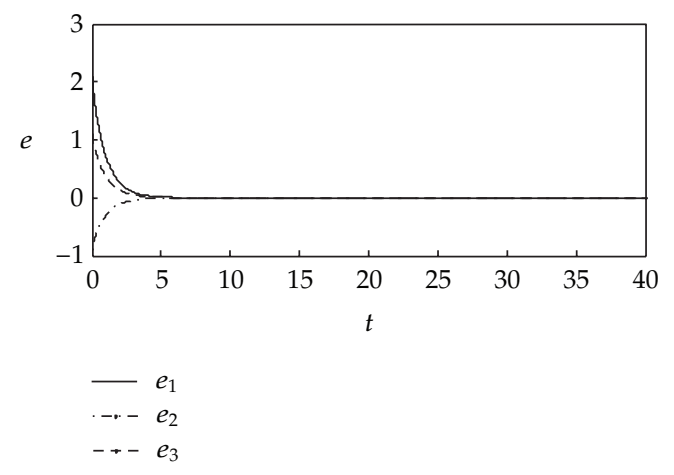

Figure 7: Synchronization errors of drive system (5.1) and response system (5.2).

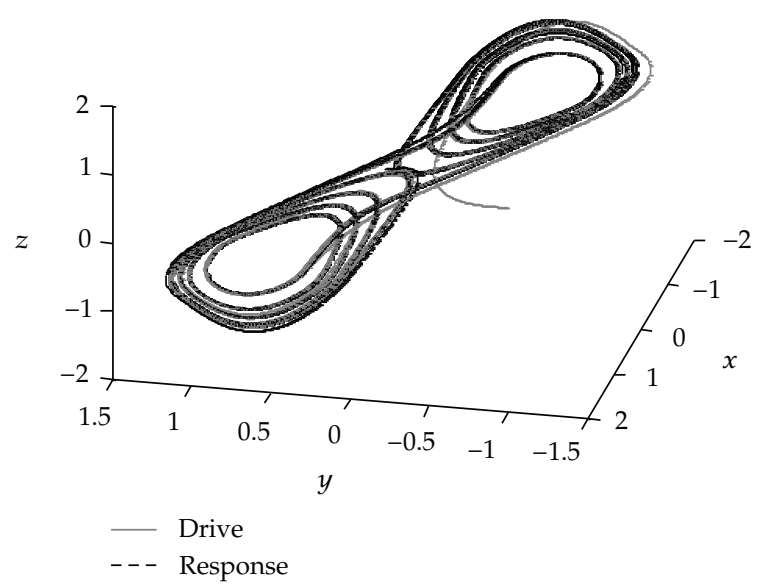

Figure 8: The attractors of drive system (5.1) and response system (5.2).

\subsection{Simulation Results}

Parameters of 3-cell CNN and Volta systems are $p_{1}=1.24, s=3.21, p_{2}=1.1, r=4.4$, and $p_{3}=1$ and $a=19, b=11$, and $c=0.73$, respectively. The initial conditions for drive and response systems are $x_{1}(0)=0.1, y_{1}(0)=0.1$, and $z_{1}(0)=0.1$, and $x_{2}(0)=8, y_{2}(0)=2$, and $z_{2}(0)=1$, respectively. By choosing $\left(y_{1}, y_{2}, y_{3}\right)=(-1,-1,-1)$, the control functions can be determined and phase synchronization between signals $\left(x_{1}, x_{2}\right),\left(y_{1}, y_{2}\right)$, and $\left(z_{1}, z_{2}\right)$ will be achieved, respectively. Numerical results are illustrated in Figures 6(a)-6(c) for fractionalorder $\alpha=0.99$. The curves of synchronization errors are shown in Figure 7, and the phase diagrams of (5.1) and (5.2) are plotted together in Figure 8.

\section{Conclusion}

This paper investigated the phase and anti-phase synchronization for the fractionalorder chaotic systems. Based on the stability criterion of the fractional-order system and tracking control, a synchronization approach is proposed. Finally, the phase and anti-phase synchronization between the fractional-order 3-cell CNN system and fractional-order Volta 
system are used to demonstrate the effectiveness of phase and anti-phase synchronization schemes.

\section{References}

[1] I. Podlubny, Fractional Differential equations, Academic Press, New York, NY, USA, 1999.

[2] R. Hilfer, Applications of Fractional Calculus in Physics, World Scientific, River Edge, NJ, USA, 2001.

[3] W. M. Ahmad and J. C. Sprott, "Chaos in fractional-order autonomous nonlinear systems," Chaos, Solitons and Fractals, vol. 16, no. 2, pp. 339-351, 2003.

[4] C. Li and G. Chen, "Chaos and hyperchaos in the fractional-order Rössler equations," Physica A, vol. 341, no. 1-4, pp. 55-61, 2004.

[5] J. G. Lü, "Chaotic dynamics and synchronization of fractional-order Arneodo's systems," Chaos, Solitons and Fractals, vol. 26, no. 4, pp. 1125-1133, 2005.

[6] D. Matignon, "Stability results for fractional differential equations with applications to control processing," in Proceedings of the Computational Engineering in Systems and Application Multiconference, pp. 963-968, IEEE-SMC, Lille, France, 1996.

[7] S. S. Delshad and M. H. Beheshti, "Generalized projective synchronization of the fractional-order hyperchaotic Lorenz systems via a vector transmitted signal," in Proceedings of the 7th International Conference on Electrical Engineering, Computing Science and Automatic Control (CCE '10), pp. 10-15, 2010.

[8] S. S. Yang and C. K. Duan, "Generalized synchronization in chaotic systems," Chaos, Solitons and Fractals, vol. 9, no. 10, pp. 1703-1707, 1998.

[9] H. J. Yu and Y. Liu, "Chaotic synchronization based on stability criterion of linear systems," Physics Letters A, vol. 314, no. 4, pp. 292-298, 2003.

[10] M. G. Rosenblum, A. S. Pikovsky, and J. Kurths, "From phase to lag synchronization in coupled chaotic oscillators," Physical Review Letters, vol. 78, no. 22, pp. 4193-4196, 1997.

[11] H. Taghvafard and G. H. Erjaee, "Phase and anti-phase synchronization of fractional order chaotic systems via active control," Communications in Nonlinear Science and Numerical Simulation, vol. 16, no. 10, pp. 4079-4088, 2011.

[12] W. H. Deng and C. P. Li, “Chaos synchronization of the fractional Lü system,” Physica A, vol. 353, no. 1-4, pp. 61-72, 2005.

[13] W. Deng and C. Li, "Synchronization of chaotic fractional Chen system," Journal of the Physical Society of Japan, vol. 74, no. 6, pp. 1645-1648, 2005.

[14] S. Bhalekar and V. Daftardar-Gejji, "Fractional ordered Liu system with time-delay," Communications in Nonlinear Science and Numerical Simulation, vol. 15, no. 8, pp. 2178-2191, 2010.

[15] P. L. Butzer and U. Westphal, "An introduction to fractional calculus," in Applications of Fractional Calculus in Physics, R. Hilfer, Ed., pp. 1-85, World Scientific, Singapore, 2000.

[16] K. S. Miller and B. Ross, An Introduction to the Fractional Calculus and Fractional Differential Equations, Wiley-Interscience, New York, NY, USA, 1993.

[17] M. Caputo, "linear models of dissipation whose Q is almost frequency independent-2," Geophysical Journal of the Royal Astronomical Society, vol. 13, no. 3, p. 529, 1967.

[18] K. Diethelm, N. J. Ford, and A. D. Freed, "A predictor-corrector approach for the numerical solution of fractional differential equations," Nonlinear Dynamics, vol. 29, no. 1-4, pp. 3-22, 2002.

[19] L. O. Chua and L. Yang, "Cellular neural networks: theory," IEEE Transactions on Circuits and Systems, vol. 35, no. 10, pp. 1257-1272, 1988.

[20] P. Arena, R. Caponetto, L. Fortuna, and D. Porto, "Bifurcation and chaos in noninteger order cellular neural networks," International Journal of Bifurcation and Chaos in Applied Sciences and Engineering, vol. 8, no. 7, pp. 1527-1539, 1998.

[21] T. T. Hartley, C. F. Lorenzo, and H. K. Qammer, "Chaos on a fractional Chua's system," IEEE Transactions on Circuits and Systems Theory and Applications, vol. 42, no. 8, pp. 485-490, 1995.

[22] I. Petráš, "Chaos in the fractional-order Volta's system: modeling and simulation," Nonlinear Dynamics, vol. 57, no. 1-2, pp. 157-170, 2009.

[23] I. Petráš, "A note on the fractional-order Volta's system," Communications in Nonlinear Science and Numerical Simulation, vol. 15, no. 2, pp. 384-393, 2010. 


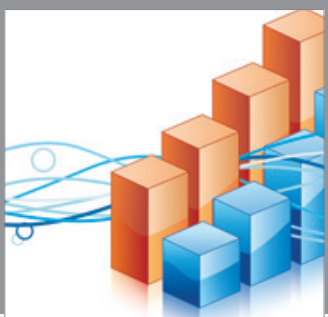

Advances in

Operations Research

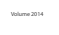

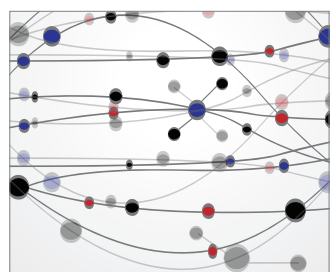

\section{The Scientific} World Journal
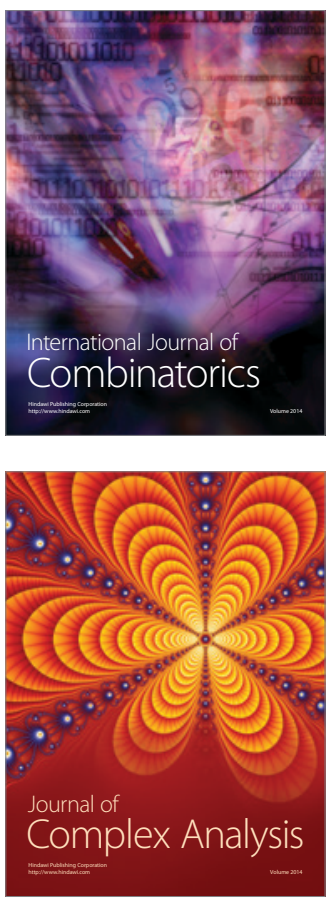

International Journal of

Mathematics and

Mathematical

Sciences
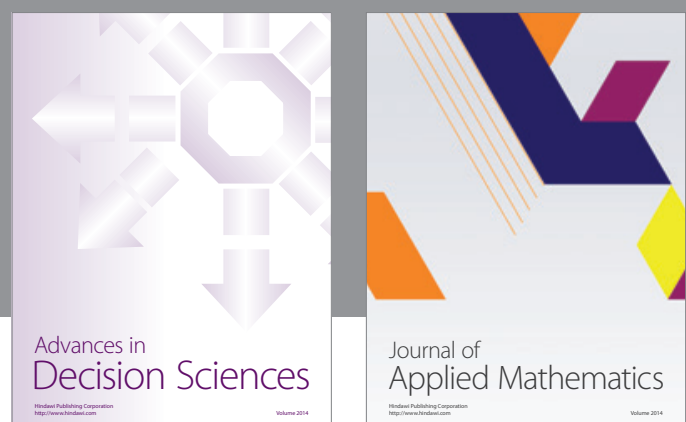

Journal of

Applied Mathematics
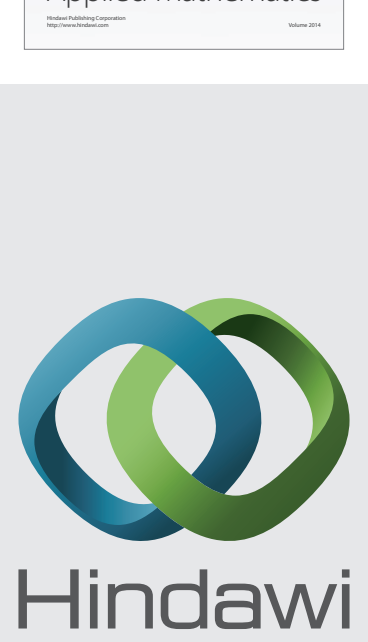

Submit your manuscripts at http://www.hindawi.com
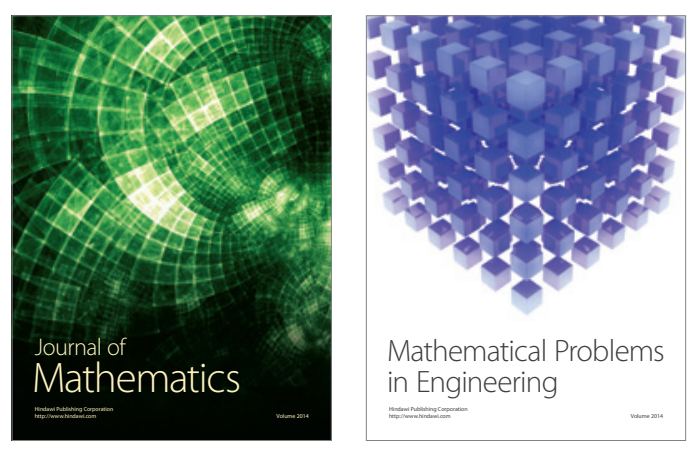

Mathematical Problems in Engineering
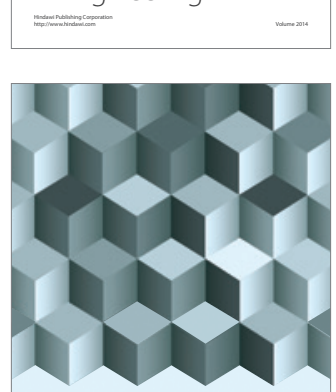

Journal of

Function Spaces
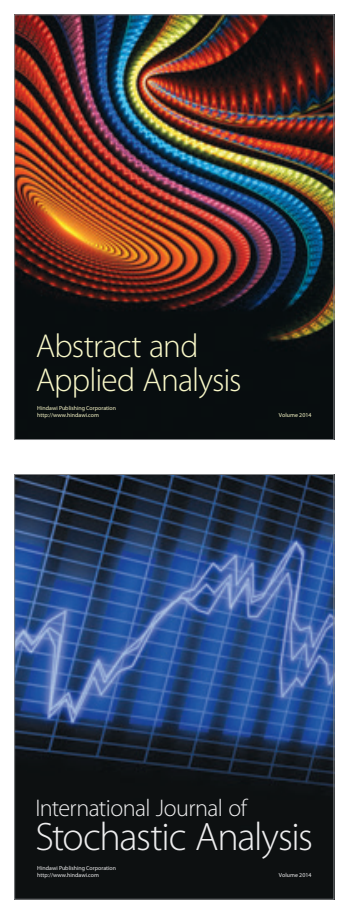

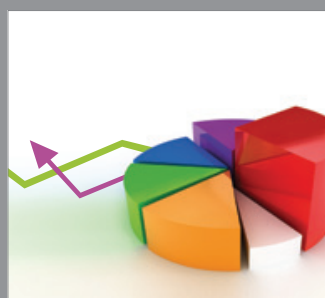

ournal of

Probability and Statistics

Promensencen
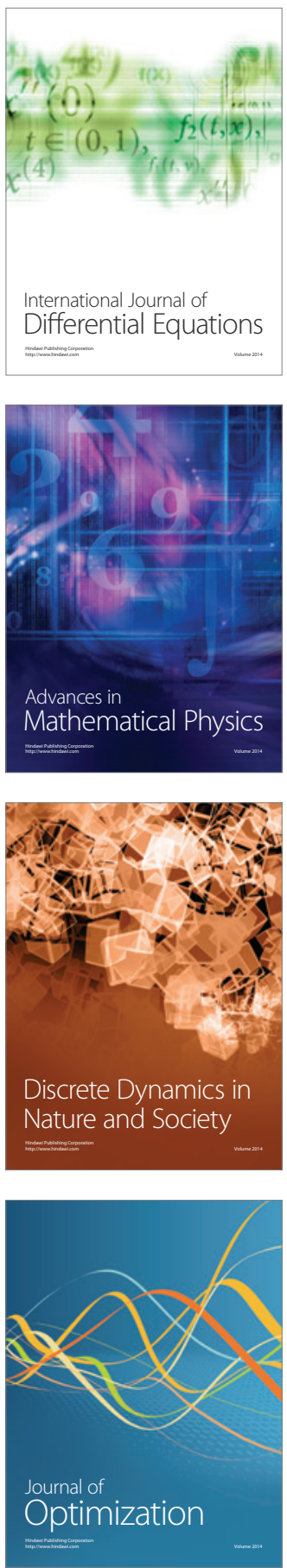